\title{
A importância dos dados para o jornalismo local ${ }^{1}$
}

Damian Radcliffe

Professor de Jornalismo na Universidade de Oregon e Pesquisador Honorário da Universidade de Cardiff.

E-mail: damianr@uoregon.edu

Resumo: $O$ analista digital, consultor, jornalista e pesquisador Damian Radcliffe é professor de Jornalismo na Universidade de Oregon, fellow do Tow Center para Jornalismo Digital na Universidade de Columbia, e Pesquisador Honorário na Escola de Jornalismo, Mídia e Cultura da Universidade de Cardiff (País de Gales). Suas pesquisas se concentram no uso de mídias sociais e tendências mais amplas na mídia local, na tecnologia, no negócio de mídia e na inovação jornalística. Neste artigo, ele defende que o jornalismo de dados pode gerar grande impacto em nível local, e embora esse tipo de comunicação seja menos dominante do que em nível nacional, existem diversos exemplos e oportunidades de boas práticas que devem ser observadas.

Palavras-chave: Damian Radcliffe; jornalismo local; jornalismo de dados; mídia local.
Abstract: A digital analyst, consultant, journalist and researcher, Damian Radcliffe is Professor in Journalism at University of Oregon, a fellow of the Tow Center for Digital Journalism at Columbia University, and an honorary research fellow at Cardiff University's School of Journalism, Media and Culture Studies (in Wales). His research focuses on the usage of social media and wider trends in local media, technology, the business of media, and journalistic innovation. In this article Radcliffe claims that data journalism can cause a great impact at a local level, and although this type of communication is less prevalent than the one at national level, there are several examples and opportunities to put it in good use that should be noted.

Keywords: Damian Radcliffe; local journalism; data journalism; local media.

\section{INTRODUÇÃO}

Os dados estão por toda parte. Big data. Little data. Dados hiperlocais. A quantidade de informação que é criada - e cada vez mais publicada - sobre nós e sobre nosso meio vem crescendo exponencialmente. Agências governamentais, empresas comerciais (tais como varejistas), mecanismos de busca e redes sociais agora geram - e têm acesso - a quantidades substanciais de dados valiosos sobre nosso comportamento, preferências e localização geográfica. As implicações disso para os cidadãos e consumidores são relevantes, mas para os jornalistas este volume de dados gerados configura-se como um benefício em potencial, criando oportunidades de storytelling e de avaliação pública em um nível nunca antes possível.

Recebido: 30/06/2016

Aprovado: 15/09/2016

1. Tradução de Mariane Murakami. 
Normalmente, muito desse esforço de criação de dados possui uma forte dimensão internacional ou nacional, com publicações como The Guardian e ProPublica, colocando-se entre os principais exemplos de conteúdo originado a partir de investigações, visualizações e dados. No entanto, como foi apontado em um projeto recente da Universidade de Westminster, que explorou o poder da mídia da pluralidade: “... é em nível local que a maioria dos cidadãos interagem com hospitais, escolas, sistemas de transporte, com a polícia e com representantes eleitos do governo" 2 .

Como resultado, a importância tanto do jornalismo local quanto do jornalismo local de dados não deve ser esquecida. Afinal de contas, esse tipo de comunicação pode fornecer informações mais significativas para o dia a dia dos leitores do que a cobertura oferecida pela mídia nacional. No entanto, no espaço dos dados, a criação de conteúdo localizado em profundidade nem sempre é fácil de fazer. Os conjuntos de dados são geralmente menores, o que acaba tornando as suas histórias menos óbvias; além disso, a maioria das redações locais operam com muito menos recursos do que as nacionais e internacionais. Esses fatores ajudam a explicar por que existe menos jornalismo de dados em nível local e porque a maior parte da análise desta atividade tanto no âmbito acadêmico quanto profissional - está focada no jornalismo de dados em nível nacional.

No entanto, é visível o valor jornalístico, público e cívico que o jornalismo local de dados pode fornecer quando bem-feito. Como resultado, esperamos que este tipo de comunicação orientada por dados torne-se mais predominante num futuro próximo. Com esse objetivo em mente, este texto apresenta uma série de estudos de caso - bem como vários princípios gerais —, concebidos para ajudar e inspirar estudantes de jornalismo, jornalistas locais, editores comunitários e profissionais de mídia hiperlocal a fazer jornalismo de dados em nível local uma realidade mais predominante.

\section{JORNALISMO HIPERLOCAL DE DADOS: CINCO ESTUDOS DE CASO DOS ESTADOS UNIDOS E DO REINO UNIDO}

Grande parte da mídia dos Estados Unidos possui raízes locais e regionais bem estabelecidas. Portanto, não é surpresa que inúmeros sites norte-americanos tenham implementado o jornalismo de dados para ajudar a contar histórias das comunidades locais a que servem. Historicamente, isso tem se concentrado principalmente na utilização de estatísticas públicas na narração de histórias relacionadas às notícias populares locais, tais como sobre crimes, saúde pública e sobre educação. Esta abordagem continua a ser bastante difundida, mas cada

2. UNIVERSITY OF WESMINSTER (ND). 'Local and Hyperlocal', blog. vez mais vemos editores utilizando dados de maneiras mais sofisticadas, tais como mapeamento, crowdsourcing e outros esforços de visualização, que vêm se tornando muito comuns. 


\subsection{Bay Citizen Bike Tracker ${ }^{3}$}

Na Califórnia, a The Bay Citizen, uma organização de notícias sem fins lucrativos, não partidária e mantida por associados, que cobre a área da Baía de São Francisco (EUA), teve sucesso ao utilizar dados públicos na produção de um rastreador de acidentes de bicicletas capaz de salvar vidas. Publicado em 2011, o microsite interativo baseou-se em cinco anos de dados para mostrar a localização de acidentes de bicicleta em toda a área da baía. Ao fazer isso, o mapa lançou mão de informações de 14.113 incidentes isolados que ocorreram entre janeiro de 2005 a dezembro de 2009, permitindo que ciclistas pudessem, assim, determinar as rotas mais seguras para percorrer e aquelas que deveriam ser evitadas.

Juntamente à utilização de resultados altamente relevantes sobre acidentes, os usuários deste serviço também podiam filtrar os dados por condições de estrada, iluminação e por outros requisitos, tais como "de quem é a culpa", produzindo, assim, um olhar com mais nuances para as causas desses acidentes. $\mathrm{O}$ site também permitia que os ciclistas e motoristas enviassem seus próprios dados sobre acidentes, uma importante contribuição a esse conjunto de dados, uma vez que a polícia só reporta acidentes quando uma ambulância é acionada. De acordo com Zusha Elinson, um dos responsáveis sobre o projeto, "nossa esperança é que isso, combinado às estatísticas da polícia, irá proporcionar uma melhor ideia de onde e por que acidentes ocorrem"4.

Blogueiro e ciclista entusiasta, Steven Vance é apenas uma das pessoas que foram inspiradas pela abordagem do The Bay Citizen; ele utilizou seu modelo para produzir um mapa semelhante para a cidade de Chicago. Mapeou os 4.931 acidentes de bicicleta relatados ao Departamento de Transportes de Illinois, ocorridos entre 2007 e 2009, 12 deles fatais ${ }^{5}$. Outras áreas onde serviços interativos semelhantes foram criados incluem Boston, Londres, Los Angeles, Nova York e Seattle.

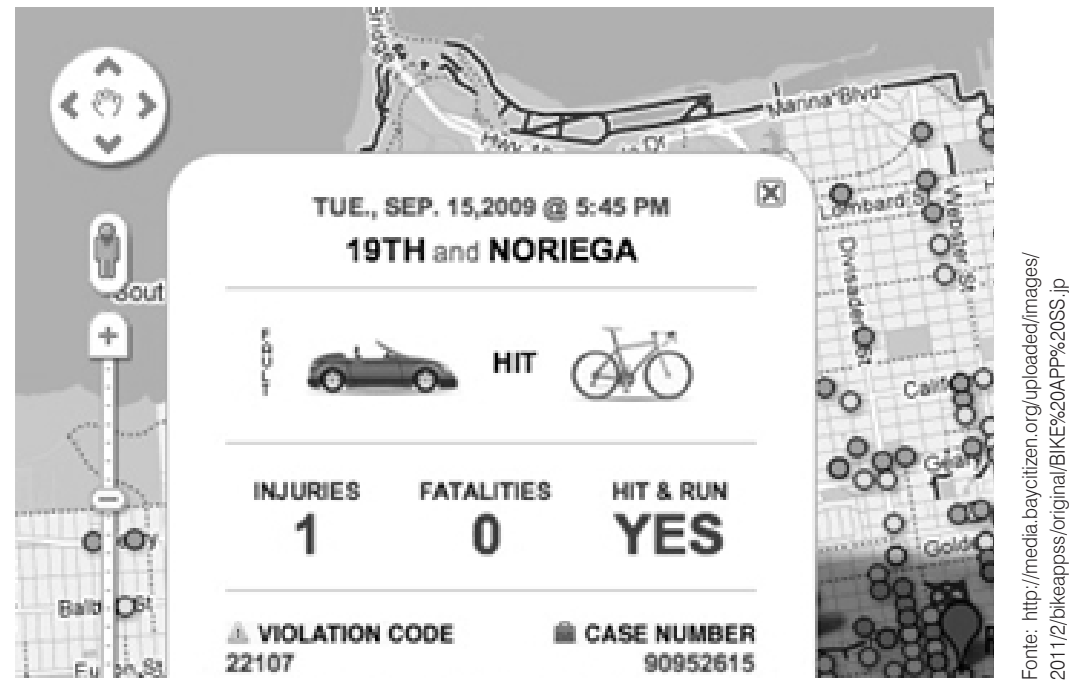

Figura 1: Captura de tela do Bike Tracker
3. O site Bay Citizen Bicycle Tracker está disponível em: <www. baycitizen.org/data/bikes/ bike-accident-tracker>.

4. ELINSON, Zusha. Police refuse to write reports for many San Francisco bike crashes. In: The Bay Citizen, 9 fev. 2011.

5. O site de Steve Vance está disponível em: <www.stevevance. net/crashportal/?page $=$ bikecrash>. 


\subsection{Programa de desligamento de iluminação de San Jose}

O site NeighborWebSJ, de San Jose (EUA), é outro caso de utilização de grande número de dados em sua produção. Embora o site esteja desativado sem nova atividade desde maio de 2014 — , ele apresentou mapas digitais para gerar informações sobre o Programa de Desligamento de Iluminação Pública, uma iniciativa de redução de custos da prefeitura e que tentava manter algumas luzes permanentemente desligadas em toda a cidade. A proposta fazia parte de um esforço mais amplo para reduzir o déficit orçamentário de US\$ 90 milhões, antecipando ainda uma redução da conta de energia elétrica anual de US\$ 3,5 milhões para USD $\$ 77,000$.

Apesar da necessidade de redução de custos, muitos residentes e empresas protestaram contra a medida, argumentando que ela aumentava o risco de crimes. Em apenas uma via, a Alum Rock Avenue, um morador reclamou que luzes estavam apagadas em treze pontos de ônibus, aumentando assim a vulnerabilidade dos passageiros. Em resposta às preocupações da comunidade, o site incluiu um mapa do Google indicando onde as luzes estavam desligadas em toda a cidade, bem como informações sobre como entrar em contato com as autoridades sobre a questão ${ }^{6}$. Essa combinação de divulgação e facilitação da comunidade ajudou a identificar que alguns postes que na verdade haviam sido desligados por acidente, enquanto outros foram ligados novamente, por conta da pressão da opinião pública (incluindo a iluminação dos pontos de ônibus da Alum Rock Avenue). Em fevereiro de 2013, a cidade concordou em religar 900 postes que tinham sido desligados como parte dos cortes orçamentais de 2008 e $2009^{7}$.

6. ROMBECK, Janice (ND a). Shutoff Streetlights Worry SJ neighborhoods. In: NeighborWebSJ.

7. ROMBECK, Janice. (ND b). 900 Streetlights Shut Off to Save Energy Costs will Shine Again Starting in March. In: NeighborWebSJ, 19 fev. 2013.

8. O mapa de homicídios de San Jose está disponível em: <www. google.com/maps/d/ viewer? $\|=37.321732 \% 2 \mathrm{C}$ $-121.875501 \&$ spn $=0.17119$ $1 \% 2 \mathrm{C} 0.205865 \& \mathrm{hl}=\mathrm{en} \& \mathrm{t}=$ $m \& m s a=0 \&$ source $=$ embe $\mathrm{d} \& \mathrm{ie}=U T \mathrm{TF} 8 \mathrm{mid}=\mathrm{z} 41 \mathrm{BAU}$ CJvuoU.kSItz3XWQv30>.

9. HOMICIDE WATCH DC. Disponível em: <http:// homicidewatch.org>.

10. ONLINE NEWS ASSOCIATION. Online Journalism Awards Winners Announced.

\subsection{Homicide Watch}

A equipe da NeighborWebSJ também produziu um mapa do Google que identificou todos os 2.012 homicídios ocorridos em San Jose, utilizando dados da imprensa policial e links para outras mídias ${ }^{8}$. Crimes sempre são furos jornalísticos, mas a fusão de dados com ferramentas interativas on-line de publicação levou o jornalismo para outro nível, até mesmo gerando seu próprio gênero com sites especializados de homicídios surgindo em diversas grandes cidades dos EUA.

Desses sites, sem dúvida o mais conhecido foi o Homicide Watch DC, que cobria assassinatos ocorridos no Distrito de Columbia. Embora o site tenha sido fechado em 31 de dezembro de 2014, ele passou seus últimos quatro anos misturando dados com relatórios e documentos judiciais originais, conteúdo de mídias sociais e colaborações de vítimas e amigos, família e vizinhos dos suspeitos, bem como qualquer informação relevante, num esforço de levar todos os homicídios do crime à convicção" ". O site foi agraciado com o Prêmio Knight Public Service pela Online News Association em 2012 ${ }^{10}$, em reconhecimento aos seus esforços de explorar uma única questão em uma única área geográfica, 
além de ter inspirado uma série de serviços semelhantes, tais como o Homicide Watch Trenton, o Homicide Watch Chicago e o The Counted, um projeto crowd sourced - mais especificamente alimentada por dados - do the Guardian "que trabalha para contar o número de pessoas assassinadas pela polícia e por outros agentes da lei nos Estados Unidos ao longo de 2015, a fim de monitorar sua demografia e contar histórias de como elas morreram"11.

\subsection{Rotas de degelo em Bournville}

Esses sites, muitos valiosos à comunidade, tais como o Homicide Watch DC — assim como vários outros aqui citados — claramente refletem a difícil realidade econômica que as operações midiáticas enfrentam, especialmente em nível local. Fazer com que o jornalismo seja rentável é um grande desafio para muitas editoras, mesmo quando elas claramente entregam valor público e conteúdo que informa e faz diferença nas comunidades. Há poucas saídas imunes a essas pressões, embora alguns sites hiperlocais, especialmente aqueles construídos por voluntários, nem sempre sofram o mesmo impacto. Os canais ultralocais também podem gerar valor ao utilizar, em conjunto ao conteúdo original, informação que já é de domínio público, reaproveitando-a de modo que seja mais útil para o público local. A abordagem pode ser particularmente útil para dados sobre saúde, criminalidade e transporte - com este último sendo um fator-chave para o consumo de mídia local.

O editor acadêmico e hiperlocal Dave Harte foi uma das pessoas que reconheceu esse potencial. Em 2010, ele produziu um mapa de degelo das estradas de Bournville ${ }^{12}$, utilizando informações disponíveis publicamente, mas apresentando-as em um formato mais user-friendly. Em seu blog, ele explicou o "processo tedioso de criação do mapa e porque os blogs hiperlocais estão condenados ao fracasso, a menos que tenhamos disponível uma fonte rica de dados locais para os alimentarmos"13. Entretanto, apesar desse tédio, ele também entendeu que tal resultado pode ser benéfico para o seu público:

Eu pensei que a escassez de areia ${ }^{14}$ poderia significar que algumas estradas não seriam degeladas caso o período de frio continuasse, e saber quais estradas receberiam esse serviço seria uma informação muito útil. "Será que a minha estrada vai ser degelada?” É uma pergunta fácil de responder uma vez que a Prefeitura possui uma lista alfabética de todas as estradas que são degeladas em ordem de prioridade $^{15}$.

No final do ano, James Cousins, vereador do partido Conservador de Shaftesbury Ward em Wandsworth (Londres), realizou algo semelhante, ao traçar a localização de todas as caçambas de areia do bairro em um mapa do Google, postando essas informações em seu $b \log ^{16}$. Os dados, antes disponíveis apenas em formato de texto - e com navegação de apenas quatro cliques - no site da prefeitura, tornaram-se muito mais acessíveis e valiosos para o público local ${ }^{17}$.
11. THE GUARDIAN. The Counted: About the Project.

12. As rotas de degelo de Bournville está disponível e m: <www.google. $\mathrm{com} / \mathrm{maps} / \mathrm{d} / \mathrm{viewer}$ ? $\mathrm{mid}=z \mathrm{FcpZs} 47 \mathrm{iTHE}$. kpKEXjMO-pYw\&hl=em>.

13. HARTE, David. Data is the New Grit. Disponivel em: <http://daveharte. com blog>. Acesso em: 14 jan. 2012.

14. Mistura de areia e sal jogada nas estradas para derreter a neve durante $\mathrm{o}$ inverno. (N. T.)

15. HARTE, David, op. cit. 16. O mapa de James Cousins está disponível em: <http://jamescousins. com/2010/12/grittingwandsworth>.

17. DALE, Robert. Engaging the Local Population - Online'. In: BBC Online, 19 out. 2011. 
comunicação \& educação • Ano XXII • número 1 • jan/jun 2017

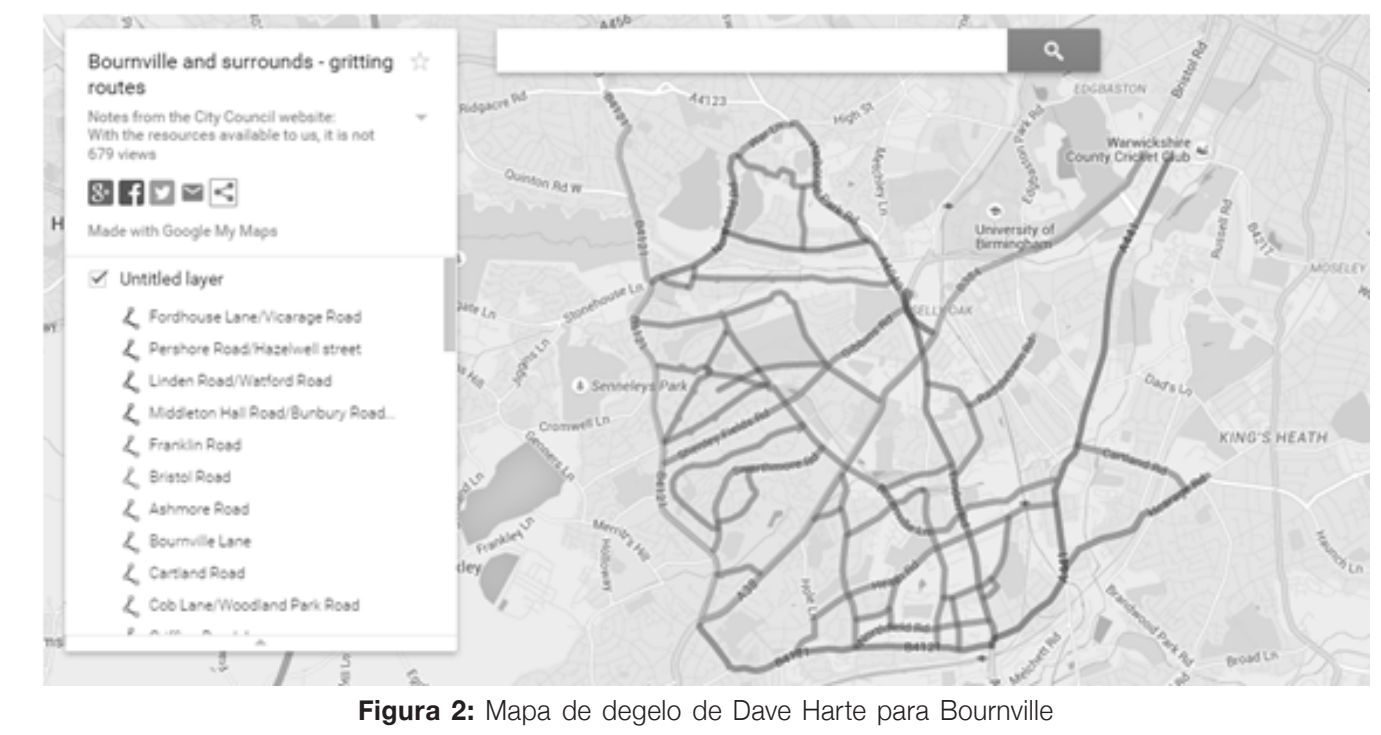

\subsection{Combatendo pontos críticos de acidente em Bramcote}

Além da apresentação de dados de formas novas e criativas, sites hiperlocais e geograficamente focados em comunidades também podem incentivar a criação de novos bancos de dados, cobrindo assuntos sobre os quais não existem dados ou que não fornecem o nível de detalhe que as comunidades e líderes locais necessitam. Este tipo de crowdsourcing pode ser observado na resposta do site NeighborWebSJ em relação ao Programa de Desligamento de Iluminação de San Jose e também na oportunidade que o público teve de contribuir para o The Bay Citizen's Bike Accident Tracker. É uma abordagem que também aparece nos resultados do Bramcote Today, sobre as discussões on-line de Hillside $\operatorname{Road}^{18}$ - um notável ponto crítico de acidentes nas periferias de Nottingham.

Como resultado dessas conversas on-line, o conselho do município instalou equipamentos para monitorar o número e a velocidade dos veículos, compartilhando os resultados ${ }^{19}$ com os leitores do Bramcote Today e envolvendo-os em uma discussão acerca de possíveis soluções, tal como um esquema de vigilância de velocidade (Austin, 2011). Algumas das medidas

18. JOHNSON, Mike. Accidents on Hillside Road. In: Bramcote Today, 14 mar. 2011a.

19. JOHNSON, Mike Accidents on Hillside Road - Traffic Survey Results. In: Bramcote Today, 29 mar. 2011b.

20. BECKETT, Charlie. Grassroots Networked Journalism Key to Future of Local News, says Polis director. In: Journalism. co.uk, 7 jun. 2010. foram implementadas com sucesso.

Este resultado estava de acordo com o ethos hiperlocal de William Perrin, fundador do Talk About Local. Sua empresa ajudou moradores de Bramcote a criar seu próprio site, além de trabalhar com outras comunidades para produzir resultados semelhantes. Fervoroso defensor da mídia hiperlocal e do jornalismo comunitário, Perrin descreveu sua ambição de:

[...] usar a web para conduzir pessoas em vias democráticas locais para fazer as coisas mudarem [...] [Os sites estão] aí para aumentar o envolvimento humano real no processo político. Você precisa de representantes para tomar decisões [...] mas a web pode ajudá-lo a entender melhor o que essas questões devem ser [...] nós ajudamos a aumentar a ação comunitária tradicional ${ }^{20}$. 


\section{JORNALISMO DE DADOS: CINCO DICAS, TRUQUES E CONSIDERAÇÕES FUNDAMENTAIS}

Como vimos, o jornalismo de dados — desde a captura de novos dados até a apresentação de informações existentes de formas novas e criativas - pode oferecer às comunidades informações valiosas para seu dia a dia. Aqui citamos cinco áreas sobre os quais jornalistas locais devem refletir, a fim de determinar a melhor forma de utilizar os dados (se o fizer) em seu trabalho.

\subsection{Parece-lhe correto, pessoalmente e em termos da história?}

Jornalismo de dados é um componente cada vez mais valorizado na cartilha do jornalista, sendo utilizado como ferramenta tanto para contar histórias, quanto para coletá-las. No entanto, apesar de o jornalismo de dados poder ajudar a gerar resultados valiosos, nem sempre é uma área fácil de se dedicar.

[...] você precisa ser um determinado tipo de pessoa. Politicamente, você precisa estar engajado e interessado no governo local, entender como ele funciona e ter uma motivação para se dedicar a ele. Ter todos esses fatores em combinação é algo muito raro ${ }^{21}$.

Barreiras específicas - mas não exclusivamente aplicáveis — aos editores locais incluem tempo, habilidades e formato no qual os dados estão disponíveis $^{22}$. O tempo é uma consideração particularmente importante para os editores hiperlocais, dado que raramente esses sites são executados em tempo integral (muitos profissionais bem-sucedidos mantêm outros trabalhos durante o dia). Enquanto isso, os jornalistas de muitas publicações locais encontram-se sob crescente pressão para produzir diariamente/semanalmente um volume cada vez maior de histórias. Como resultado, jornalistas e editores podem chegar à conclusão de que eles não podem cobrir tudo ${ }^{23}$ e que o seu tempo limitado é mais bem servido com foco na entrega de conteúdos de produção mais rápida (e muitas vezes mais fácil). Os jornalistas não devem, claro, ter aversão ao trabalho duro, mas eles também precisam se perguntar se a abordagem orientada por dados é o caminho correto e, se for, como utilizá-la de forma eficaz.

\subsection{Posso usar uma solução "pronta"?}

Uma forma que editores locais podem ajudar a tornar as coisas mais fáceis para si mesmos é utilizando recursos prontos - como por exemplo, o dispositivo MySociety's FixMyStreet (www.fixmystreet.com) - para ajudá-los a produzir conteúdo gerado por dados. Essa ferramenta on-line permite que as pessoas "reportem, vejam ou discutam problemas locais como pichações, descarte ilegal de lixo, calçadas ou iluminação pública quebradas ", e sites no Reino
21. WORTHY, Ben. Where are the Armchair Auditors?' Open Data Institute, 3 jun. 2013.

22. WHEELER, Brian. Government Online Data Ignored by "Armchair Auditors". BBC News, 9 nov. 2012.

23. JONES, Richard. Thirteen Lessons I've Learned from Running a Hyperlocal Site'. In: The Richard Jones Journalism Blog, 5 out. 2011. 
24. Um contador em tempo real do número total de incidentes reportados no Reino Unido está disponivel em: <https:// www.fixmystreet.com>.

25. FIXMYSTREET. FixMyStreet - Press Use.

26. GAHRAN, Amy SeeClickFix: Crowdsourced local problem reporting as community news'. In: Knight Digital Media Center, 19 set. 2012.

27. SCHILLER, citado em SONDERMAN, Jeff. NBC Closes Hyperlocal, Data-driven Publishing Pioneer. In: Poynter, 7 fev. 2013.

28. WRIGHT, Paul. EveryBlock: The Online Community for your JNeighborhood. Comcast Voices, 26 ago. 2014.

29. EveryBlock FAQ. Disponível em: <www. everyblock.com/faq $>$. Acesso em: 15 set. 2015.

30. JOHNSON, Steve. Sorry EveryBlock, You Never Learned how to Write a Headline. In: Hudson Eclectic, 8 fev. 2013.
Unido e em outros lugares têm se beneficiado com este aplicativo e plug-in on-line. Desde que foi lançado, no início de fevereiro de 2007, mais de 700 mil relatos $^{24}$ de incidentes foram feitos por meio deste serviço só no Reino Unido; a MySociety relatou que mais de $50 \%$ dos usuários nunca haviam entrado em contato com a prefeitura, mostrando que a ferramenta pode desempenhar um papel importante na promoção da cidadania ativa ${ }^{25}$, bem como ser uma fonte fértil para uma variedade de iniciativas jornalísticas.

Alguns hiperlocais dos EUA utilizam uma ferramenta semelhante, a SeeClickFix (en.seeclickfix.com) para criar ações de conteúdo e de comunidades em torno de questões que são importantes para o seu público. Os dados capturados por esses serviços podem destacar problemas, gerar histórias e promover debates. É "um exemplo de jornalismo comunitário que não necessariamente vem embalado em forma de história” ${ }^{26}$, mas que, no entanto, fornece insights importantes para as preocupações das comunidades locais.

\subsection{Lembre-se que os dados por si só não são suficientes}

Embora ferramentas como SeeClickFix e FixMyStreet - bem como plataformas públicas de dados abertos - sejam recursos extremamente úteis para os jornalistas, elas só fazem metade do trabalho. Dados por si só não contam uma história: ainda precisamos de jornalistas para decidir como organizar, analisar e visualizá-los, bem como para fornecer o contexto e interpretação apropriados. Essas considerações são importantes para editores, e o fracasso inicial do EveryBlock - um site norte-americano que agregava informações locais produzidas por agências governamentais e estaduais - apenas reforça este ponto.

$\mathrm{O}$ site, que ofereceu uma abordagem centrada em dados para o hiperlocal, foi lançado em 2008 e foi comprado pelo MNBC em 2009. No entanto, em fevereiro de 2013, o site foi fechado depois que a NBC decidiu que eles "não veem um lugar estratégico para EveryBlock dentro do portfólio [do jornalismo digital da NBC]" ${ }^{27}$. Em janeiro de 2014, com a marca EveryBlock e serviço então de propriedade da Comcast - seguindo de sua aquisição da NBCUniversal - o site foi relançado em Chicago, e depois, na Filadélfia, no final de agosto daquele $\operatorname{ano}^{28}$. Desde então, Houston, Boston e Denver foram adicionadas a esta lista de operações ativas ${ }^{29}$.

Um dos problemas originais de EveryBlock, como observou Steve Johnson, professor assistente de Jornalismo Eletrônico na Universidade Estadual de Montclair, foi: "Os leitores não se importam com dados brutos. Eles querem a história que está nos dados" ${ }^{30}$. Para ilustrar, ele explicou o que aconteceu quando explorou alguns dados relacionados à área da baixa Manhattan:

Existiam relatórios sobre quais pichações a prefeitura dizia que haviam sido apagadas a cada mês e em cada bairro. Mas estavam faltando contexto e fotos. Se eu sou um repórter escrevendo uma história sobre pichações, quero mostrar fotos com antes e depois e, mais importante, quero saber se a cidade está lutando 
com sucesso contra os pichadores, isto é, quem está ganhando. Os dados brutos não oferecem isso ${ }^{31}$.

Esta falta de contexto é, talvez, uma das razões pelas quais o site originalmente não conseguiu repercutir efetivamente entre o público ou anunciantes para sobreviver; e, apesar de sua recente ressurreição, esta experiência decepcionante oferece lições valiosas para os editores.

\section{4 $O$ valor do jornalismo em rede}

Até certo ponto, a equipe EveryBlock reconheceu as deficiências da sua abordagem em que colocava os dados em primeiro lugar quando, em 2011, o site mudou-se para uma nova direção, dizendo a seu público:

Por mais valiosas que as atualizações automáticas sobre crime, menções de mídia e outras notícias do EveryBlock sejam, as contribuições de amigos e vizinhança tornam-se consideravelmente mais significativas e úteis. Ainda que nós não estejamos removendo a nossa coletânea existente de registros públicos e de outras informações sobre o bairro (mais sobre isso mais tarde), nós percebemos que a participação humana é essencial, não apenas como um complemento, mas como o alicerce do site $e^{32}$.

Antes do seu ressurgimento em 2014, pela Comcast, esta tentativa foi inicialmente malsucedida, destacando a importância - na produção de notícias e informação sobre a comunidade - de envolver e engajar a comunidade neste processo desde o início. Técnicas para fazer isso incluem crowdsourcing de informação, verificação de fatos e aproveitamento de conhecimentos específicos do público, como fez o site de Birmingham Help me Investigate (helpmeinvestigate. com), numa base caso a caso.

Trabalhar dessa forma pode exigir uma mudança de abordagem de algumas editoras. Mas como argumentou o professor Jeff Jarvis:

Profissional e amador, jornalista e cidadão podem agora trabalhar em conjunto para reunir e compartilhar mais notícias, de mais maneiras e para mais pessoas como jamais foi possível antes. Jornalismo em rede baseia-se em uma verdade simples, autoevidente e autointeressada: nós podemos fazer mais juntos do que separados... Isto, creio eu, é o estado natural dos meios de comunicação: bidirecional e colaborativo ${ }^{33}$.

\subsection{Determine sua abordagem em base caso a caso}

O jornalismo de dados pode estar em voga no momento, mas seu uso deve ser avaliado como qualquer outra potencial abordagem editorial, com jornalistas determinando se, quando e como, o uso de ferramentas e técnicas de jornalismo de dados agregam valor às histórias que estão sendo contadas. O sentimento é tão aplicável nas notícias e informações locais, quanto é na
31. Idem, ibidem

32. HOLOVATY, Adrian. EveryBlock's First Major Redesign. EveryBlock blog, 21 mar. 2011.

33. JARVIS, Jeff. Supermedia: Saving Journalism So It Can Save the World. In: BuzzMachine, 6 jun. 2008. 
cobertura de eleições, na elaboração de relatórios sobre a despesa pública ou na cobrança da prestação de contas de instâncias nacionais. Quando bem utilizada, a mídia local pode aproveitar o jornalismo de dados para informar o público, apoiar campanhas e angariar novos insights sobre o interesse do seu público.

Ao fazer isso, muitos editores estão fazendo uso extensivo de mapas e ferramentas de mapeamento, abraçando widgets prontos e capturando dados que ajudam a complementar seus relatórios e campanhas. Eles também estão apresentando dados de forma ainda mais visualmente interessante - veja, por exemplo, o uso de mapas e infográficos do site DNAinfo.com, de Nova York, na demonstração da sua análise de números de blitz e revista policial no ano de $2011^{34}$.

Em consonância com as preferências da audiência por conteúdo mais visual, empresas de mídia também estão criando mais aplicativos e microsites orientados por dados, proporcionando uma experiência de imersão muito detalhada - como nunca antes possível - em assuntos como acidentes de bicicleta, despesas de órgãos governamentais ou cortes no setor público ${ }^{35}$. O fato de que cada história possa ser contada de uma maneira diferente é um lembrete valioso que nem todo o jornalismo pode ser produzido com padrões.

Como Simon Rogers argumentou, devido à sua natureza "o jornalismo de dados é um grande nivelador [...] muitos grupos de mídia estão começando com tanto conhecimento e experiência quanto alguém trabalhando de seu quarto" ${ }^{36}$. Isto é especialmente verdadeiro no âmbito do "dado pequeno", em que há uma margem considerável para comunicação baseada em dados, bem como para um maior espaço para inovação e criatividade. Essas abordagens devem depender de uma combinação entre: a história sendo contada, as habilidades dos jornalistas envolvidos, a necessidade e vontade do público, bem como os meios utilizados. Como este trabalho mostra, existem muitas maneiras de se fazer isso; esse acontecimento depende, agora, dos editores e dos jornalistas locais.

\section{REFERÊNCIAS BIBLIOGRÁFICAS}

AUSTIN, Steve. 'Hillside Road Proposals' in Bramcote Today, 4 de abril, 2011. Disponível em: <http://bramcotetoday.org.uk/2011/04/04/hillside$\mathrm{road} />$. Acesso em: 6 out. 2013.

BECKETT, Charlie. Grassroots Networked Journalism Key to Future of Local News, Says Polis Director. In: Journalism.co.uk, 7 jun. 2010. Disponível em: $<$ www.journalism.co.uk/news-features/grassroots-networked-journalism-keyto-future-of-local-news-says-polis-director/s5/a539020>. Acesso em: 6 out. 2013. 
BRADSHAW, Paul. 'Announcing Help Me Investigate: Networks' in Online Journalism Blog, 7 de novembro, 2011. Disponível em: <http:// onlinejournalismblog.com/2011/11/07/announcing-help-me-investigatenetworks>. Acesso em: 6 out. 2013.

COLVIN, Jill and Davis, Paul. 'Port Authority is top stop-and-frisk hotspot regardless of race', in DNAinfo, New York, 4 de junho, 2012. Disponível em: $<$ www.dnainfo.com/new-york/20120604/new-york-city/port-authority-is-topstop-and-frisk-hotspot-regardless-of-race\#ixzzlwrTXMVW>. Acesso em: 6 out. 2013.

DALE, Robert. Engaging the Local Population - Online. In: BBC Online, 19 out. 2011. Disponível em: <www.bbc.co.uk/blogs/blogcollegeofjournalism/ posts/engaging_the_local_population>. Acesso em: 6 out. 2013.

ELINSON, Zusha. Police refuse to write reports for many San Francisco bike crashes. In: The Bay Citizen, 9 fev. 2011. Disponível em: <www.baycitizen.org/ news/bikes/police-refuse-reports-bike-accidents>. Acesso em: 14 set. 2015.

FIXMYSTREET. FixMyStreet - Press Use. Disponível em: <www.mysociety. org/press-area/fixmystreet>. Acesso em: 6 out. 2013.

GAHRAN, Amy. 'SeeClickFix: Crowdsourced local problem reporting as community news. In: Knight Digital Media Center, 19 set. 2012. Disponível em: $<$ www.knightdigitalmediacenter.org/blogs/agahran/2012/09/seeclickfixcrowdsourced-local-problem-reporting-community-news $>$. Acesso em: 6 out. 2013.

HARTE, David. Data is the New Grit. In: <http://daveharte.com> blog. Acesso em: 14 jan. 2012. Disponível em: <http://daveharte.com/bournville/data-isthe-new-grit>. Acesso em: 6 out. 2013.

HOLOVATY, Adrian. EveryBlock's First Major Redesign. EveryBlock blog, 21 mar. 2011. Disponível em: <http://blog.everyblock.com/2011/mar/21/ redesign>. Acesso em: 6 out. 2013.

HOMICIDE WATCH DC. Disponível em: <http://homicidewatch.org $>$. Acesso em: 14 set. 2015.

HOUSE OF COMMONS CULTURE, Media and Sport Committee (2010) Future for local and regional media, House of Commons, London, 24 de março, 2010. Disponível em: <www.publications.parliament.uk/pa/ cm200910/cmselect/cmcumeds/43/4302.htm>. Acesso em: 6 out. 2013.

JARVIS, Jeff. Supermedia: Saving Journalism So It Can Save the World. In: BuzzMachine, 6 jun. 2008. Disponível em: <http://buzzmachine. com/2008/06/06/supermedia>. Acesso em: 6 out. 2013. 
comunicação \& educação • Ano XXII • número 1 • jan/jun 2017

JOHNSON, Mike. Accidents on Hillside Road. In: Bramcote Today, 14 mar. 2011a. Disponível em: <http://bramcotetoday.org.uk/2011/03/14/ accidents-on-hillside-road>. Acesso em: 6 out. 2013.

Accidents on Hillside Road - Traffic Survey Results. In: Bramcote Today, 29 mar. 2011b. Disponível em: <http://bramcotetoday.org. uk/2011/03/29/hillside-road-survey-results-2>. Acesso em: 6 out. 2013.

JOHNSON, Steve. Sorry EveryBlock, You Never Learned how to Write a Headline. In: Hudson Eclectic, 8 fev. 2013. Disponível em: <http:// hudsoneclectic.com/2013/02/08/sorry-everyblock-you-never-learned-howto-write-a-headline>. Acesso em: 6 out. 2013.

JONES, Richard. Thirteen Lessons I've Learned from Running a Hyperlocal Site. In: The Richard Jones Journalism Blog, 5 out. 2011. Disponível em: $<$ http:/ / richardjonesjournalist.com/2011/10/05/thirteen-lessons-ivelearned-from-running-a-hyperlocal-site>. Acesso em: 6 out. 2013.

ONLINE NEWS ASSOCIATION. Online Journalism Awards Winners Announced. Disponível em: <http://journalists.org/2012/09/24/2012online-journalism-award-winners-announced>. Acesso em: 14 set. 2015.

ROGERS, Simon. Anyone can do it: data journalism is the new punk. Guardian Datablog, 24 de maio, 2012. Disponível em: <www.theguardian.com/news/ datablog/2012/Maio/24/data-journalism-punk>. Acesso em: 14 set. 2015.

ROMBECK, Janice. (ND a). Shutoff Streetlights Worry SJ Neighborhoods. In: NeighborWebSJ. Disponível em: <www.neighborwebsj.com/shutoffstreetlights-worry-sj-residents>. Acesso em: 6 out. 2013.

(ND b). 900 Streetlights Shut Off to Save Energy Costs will Shine Again Starting in March. In: NeighborWebSJ, 19 fev. 2013. Disponível em: $<$ www.neighborwebsj.com/900-streetlights-shut-off-to-save-energy-costs-willshine-again-starting-in-March>. Acesso em: 6 out. 2013.

SONDERMAN, Jeff. NBC Closes Hyperlocal, Data-driven Publishing Pioneer. In: Poynter, 7 fev. 2013. Disponível em: <www.poynter.org/latest-news/topstories /203437/nbc-closes-hyperlocal-pioneer-everyblock>. Acesso em: 6 out. 2013.

THE GUARDIAN. The Counted: About the Project. Disponível em: <www. theguardian.com/us-news/ng-interactive/2015/jun/01/about-thecounted>. Acesso em: 14 set. 2015.

UNIVERSITY OF WESMINSTER (ND). Local and Hyperlocal blog. Disponível em: <http://www.mediaplurality.com/local-and-hyperlocal>. Acesso em: 14 set. 2014. 
WATT, Andrew. 'Birmingham budget cuts ... The story so far', in Watt's Going On, 20 de novembro, 2010. Disponível em: <http:/ / watts-going-on.blogspot. com/2010/11/birmingham-budget-cuts-story-so-far.html $>$. Acesso em: 6 out. 2013.

WHEELER, Brian. Government Online Data Ognored by Armchair Auditors. In: BBC News, 9 nov. 2012. Disponível em: <www.bbc.co.uk/news/ukpolitics-20221398>. Acesso em: 6 out. 2013.

WRIGHT, Paul. EveryBlock: The Online Community for your Neighborhood. In: Comcast Voices, 26 ago. 2014. Disponível em: <http://corporate. comcast.com/comcast-voices/everyblock-the-online-community-for-yourneighborhood>. Acesso em: 15 set. 2015.

WORTHY, Bem. Where are the Armchair Auditors? In: Open Data Institute, 3 jun. 2013. Disponível em: <www.theodi.org/blog/guest-blog-where-arearmchair-auditors $>$. Acesso em: 6 out. 2013. 